\title{
Clinical value of oblique lateral lumbar interbody fusion combined with posterior navigation and long segment internal fixation in the treatment of degenerative scoliosis
}

\author{
Hui Fei \\ hospital \\ Feng Hong \\ hospital \\ Canfeng Wang \\ hospital \\ Renfu Quan \\ hospital
}

Yuliang Lou (D360465602@qq.com )

no https://orcid.org/0000-0001-9750-1482

\section{Research article}

Keywords: Scoliosis; Surgery,computer-assisted; Spine fusion

Posted Date: April 7th, 2020

DOI: https://doi.org/10.21203/rs.3.rs-18523/v1

License: (c) (1) This work is licensed under a Creative Commons Attribution 4.0 International License.

Read Full License 


\section{Abstract}

Background $₫ T 0$ assess the short-term clinical outcomes of oblique lateral lumbar interbody fusion (OLIF) combined with multiple level fixation with the guidance of posterior "O" arm CT navigation for degenerative scoliosis.

Methods $₫ A$ total of 15 patients ( 3 males and 12 females; average age: $62.2 \pm 5.3$ years) with degenerative scoliosis were enrolled in our study. All patients were treated with OLIF combined with posterior "O" arm CT navigation and long segment (T 10 -L 5 ) internal fixation during Apr. 2016 and Dec. 2017. Collected perioperative data included surgery time, blood loss, excellent rate of intraoperative pedicle screw. Coronal Cobb angle, lumbar lordosis (LL), intervertebral space height, sagittal balance (SVA) were measured by plain radiograph. Accuracy of pedicle screw placement and intervertebral fusion was investigated with postoperative CT. Visual analogue scale (VAS) and Oswestry disability index (ODI) were used to evaluate the clinical efficacy of surgery.

Results $₫$ All patients were followed for $12-25$ months ( $16.5 \pm 5.3$ months). The OLIF operation time was (98.7 \pm 16.8$) \mathrm{min}$, and the blood loss was $(50.2 \pm 10.7) \mathrm{ml}$. The posterior "O" arm CT-navigated long segment internal fixation was $(101.5 \pm 23.4) \mathrm{min}$, and the blood loss was $(63.1 \pm 19.7) \mathrm{ml}$. The total operation time was $(200.2 \pm 40.2) \mathrm{min}$, and the blood loss was $(113.3 \pm 30.4) \mathrm{ml}$. Coronal Cobb angle, LL, intervertebral space height, SVA, VAS, ODI at the last follow-up were $5.5^{\circ} \pm 3.1^{\circ}, 39.3^{\circ} \pm 9.1^{\circ},(10.9 \pm 1.2)$ $\mathrm{mm},(25.1 \pm 10.9) \mathrm{mm},(1.3 \pm 0.8)$ and $(15.8 \pm 4.5) \%$, respectively, showing statistically significant differences $(\mathrm{P}<0.05)$. The "O" arm CT navigation has 240 nails, and the excellent rate of three-dimensional scanning of the "O" arm during surgery is $96 \%$. Lumbar CT showed lumbar fusion at the 6-month postoperative period.

Conclusion $\triangle$ The short-term clinical outcomes of oblique lateral lumbar interbody fusion combined with posterior "O" arm CT-navigated long segment internal fixation for degenerative scoliosis are satisfactory, with advantages like minimal invasiveness, accurate navigation, high bone fusion rate and few complications. Keywords $₫$ Scoliosis; Surgery,computer-assisted; Spine fusion

\section{Introduction}

Degenerative scoliosis (DS) refers to the lateral curvature of spinal coronal plane, disappeared forward curvature of sagittal lumbar spine or segmental backward curvature that is caused by degeneration of single or multiple intervertebral discs and asymmetric degeneration of corresponding articular processes after skeletal maturation in the previous absence of spinal deformity. DS occurs frequently among people over 50 years of age, whose main clinical symptoms are refractory back pain, intermittent claudication, radiculopathic pain and progressive lateral curvature of the spine ${ }^{[1-4]}$.

Currently, conservative therapy of DS is considered to be ineffective, so surgical treatment is needed in most cases ${ }^{[5]}$. Despite preferable clinical outcomes with the conventional posterior osteotomy and orthopedic internal fixation, problems such as long operative duration, large intraoperative bleeding 
volume, high technical requirements and slow postoperative recovery are present, which can be hardly tolerable by the physical condition of middle-aged and elderly patients ${ }^{[6-9]}$.

Accordingly, the concept of minimally invasive spinal surgery has emerged. In recent years, good efficacy for DS treatment has been attained by extreme lateral interbody fusion or direct lumbar interbody fusion (XLIF/DLIF) combined with posterior internal fixation. However, the operation passes through the psoas muscle fibers, which can cause damage to the psoas muscle and lumbar plexus, with rather high neurological complications ${ }^{[10-12]}$.

Oblique lumbar interbody fusion (OLIF), as a currently popular minimally invasive procedure in spine surgery, removes intervertebral disc and fuses bone grafts through the natural gap between large abdominal vessels and psoas muscle, which causes less damage to the psoas muscle, lumbar plexus and leads to less neurological complications than XLIF/DLIF ${ }^{[13]}$. Satisfactory outcomes with OLIF have been reported by many scholars at home and abroad in the treatment of lumbar degenerative diseases [13-17]. As a novel 3D stereoscopic imaging system, "O" arm CT navigation technology is superior to conventional fluoroscopy in terms of shortening operation time, pedicle screw accuracy, and reducing radiation exposure of surgeons and patients ${ }^{[18-19]}$.

However, application of OLIF combined with posterior "O" arm CT-navigated long-segment internal fixation in DS patients has been rarely reported. Through a retrospective case series study, we analyzed the clinical data of 15 DS patients admitted for treatment in our hospital from April 2016 to December 2017, and discussed the clinical outcomes of OLIF combined with posterior "O" arm CT-navigated longsegment internal fixation for the treatment of DS, in order to provide an evidence for clinical operation.

\section{Materials And Methods}

\section{Study design}

This study was approved by the ethics committee of our hospital and all patients signed informed consent before participation.Patients who underwent an OLIF combined with posterior "O" arm CTnavigated long-segment internal fixation between April 2016 to December 2017 were include in this study.All procedures were performed by a single surgeon in our hospital . Inclusion criteria: 1) Lumbar scoliosis on the full-spine PA \& LAT X-rays, with a Cobb angle of $>10$, and location of the scoliotic apex at $\left.L_{1} \sim L_{5} ; 2\right)$ Refractory back and leg pain, poor efficacy upon 3 months of conservative treatment, and no obvious signs of neurological impairment; 3) Suitability for OLIF surgery based on the preoperative lumbar CT and MRI. Exclusion criteria: 1) Previous history of spinal surgery; 2) Other types of scoliosis, such as congenital spinal deformity and ankylosing spondylitis; 3 ) Any history of spinal tumors or tuberculosis; 4) Severe osteoporosis ( $T<-3.5$, with history of fracture of other parts) ${ }^{[20]}$.

\section{Surgical Procedure}


OLIF.A V-shape incision about $4 \mathrm{~cm}$ in length was made at the left lower abdomen. The skin, subcutaneous tissue and deep fascia were cut layer by layer, and the extra-abdominal oblique muscle, intra-abdominal oblique muscle and transverse abdominal muscle were dissected bluntly along the muscle fiber direction. The retroperitoneal space was entered, and the abdominal aorta and psoas space were exposed under direct vision. The intervertebral disc was exposed, the psoas muscle was retracted clinging to the disc surface, and the guide needles were inserted into the vertebral bodies of upper and lower segments. Then the intervertebral disc was excised and scraped to the bone endplate. The intervertebral space was dilated by sequential trials, and the artificial bone was filled with an appropriate size anterior interbody fusion cage. After initial oblique entrance, the fusion cage was placed vertically into the intervertebral space by gradual rotation, which was positioned via the "C" arm fluoroscopy.

Posterior "O" arm CT-navigated long-segment internal fixation .The $\mathrm{T}_{10} \mathrm{~L}_{5}$ spinous process connection was incised, separated and entered via the longissimus-multifidus approach to expose the spinous processes, as well as the superior and inferior articular processes. The smart surgical tool was authenticated, and the tracer was installed by choosing the $L_{2}$ spinous process. The $T_{10}{ }^{-} L_{5}$ vertebral bodies were scanned over 360 degrees by "O" arm 3D CT, and uploaded to the view base station to generate 3D images. The optimal pedicle screw insertion point was selected using the smart surgical tool with the aid of 3D navigation, and then the pedicle screws were placed sequentially according to the depth of smart probe. After completion of nailing, the $T_{10}-\mathrm{L}_{5}$ vertebral bodies were subjected to " $\mathrm{O}$ " arm $3 \mathrm{D}$ scanning to assess the screw position. After satisfactory screw fixation, the plastic connecting rod was placed and locked.

Neuroelectrophysiological monitoring (cortical somatosensory evoked potentials and motor evoked potentials) was employed throughout the surgery, and autologous blood transfusion was used.

\section{Follow-up evaluation}

\section{Surgical conditions}

Operation time: A period from surgical incision of skin to the completion of incision suturing. Intraoperative bleeding volume: Blood losses from intraoperative revealed hemorrhage concealed hemorrhage. Among them, the concealed bleeding volume was measured by the Gross equation [19] based on the height, weight and pre- and postoperative changes in hematocrit .

\section{Clinical efficacy}

Symptomatic relief in patients was assessed using the visual analogue scale (VAS) $\left.{ }^{[20}\right]$ and the Oswestry disability index (ODI) ${ }^{[21]}$.

\section{Radiograph evaluation indices}


Coronal Cobb Angle: After determining the upper and lower end vertebrae of lateral curvature, a vertical line was made each for the parallel line of upper end vertebra superior endplate and for the parallel line of lower end vertebra interior endplate, and the angle between the two vertical lines was precisely the Cobb angle. Lumbar lordosis (LL): The angle between the parallel line of $L_{1}$ vertebral body superior endplate and the parallel line of $S_{1}$ vertebral body superior endplate. Intervertebral space height: The average height value of the anterior intervertebral space margin and the posterior intervertebral space margin on the full-spine LAT X-ray. Sagittal vertical axis (SVA): The distance between the C7 plumb line and the upper back angle of $S 1$. Sagittal imbalance was defined to be $S V A>5 \mathrm{~cm}$. The excellent rate of pedicle screw position was evaluated by the Richter method ${ }^{[22]}$. Excellent: the screw was located entirely inside the pedicle and vertebral body; Good: the screw penetrated the pedicle isthmic cortex $(<1 / 4$ of screw diameter); Poor: the screw penetrated the pedicle isthmic cortex distinctly ( $>1 / 4$ of screw diameter). Intervertebral fusion rate: Assessed by the Siepe method for lumbar vertebral 3D CT reconstruction [23].

\section{Statistical analysis}

Statistical analysis was performed using SPSS 19.0 (SPSS, USA) software. The measurements were showedas Mean $\pm S D$. The pre- and postoperative VAS scores for low back and leg pain, ODI, coronal Cobb angle, LL, intervertebral space height and SVA were compared by the paired data t-test at a bilateral significance level of $a=0.05$. $P<0.05$ was considered statistically significant.

\section{Results}

Among 15 DS patients treated by OLIF combined with posterior "O" arm CT-navigated long-segment internal fixation in our hospital From April 2016 to December 2017, they consisted of 3 males and 12 females, who were aged 55-73 years old, with a mean age of $(62.2 \pm 5.3)$ years. Apical scoliotic vertebrae were $L_{2 / 3}$ in 9 cases; and $L_{3 / 4}$ in 6 cases. Preoperative bone density values ranged from 0.3 to $-1.8 \mathrm{SD}$, with an average of $(-0.8 \pm 0.4) \mathrm{SD}$. All patients suffered from refractory low back pain, 10 patients had accompanying intermittent claudication, while 5 patients had accompanying radiculopathic pain. None of them were cured effectively after 3 months of regular conservative treatment, thus affecting their quality of life. All patients received OLIF combined with posterior "O" arm CT-navigated long-segment internal fixation (lumbar fusion cage, Sanyou Medical Co., Ltd., Shanghai; Medtronic CT navigation, USA; US long arm universal screw, Fule Technology Co., Ltd., Beijing).

All patients were followed up for 12 to 25 months, with a mean of $(16.5 \pm 5.3)$ months.

\section{Surgical parameters}

All 15 patients received successful surgery, including 3 cases of double-segmental OLIF, 8 cases of threesegmental OLIF and 4 cases of four-segmental OLIF. Regarding posterior approach, all were treated by long segment $\left(T_{10}-L_{5}\right)$ internal fixation under the "O" arm CT navigation. For the OLIF, operation time was $(98.7 \pm 16.8) \mathrm{min}$, and the intraoperative bleeding volume was $(50.2 \pm 10.7) \mathrm{ml}$. For the posterior "O" arm CT- 
navigated long segment internal fixation, the operation time was $(101.5 \pm 23.4) \mathrm{min}$, and the intraoperative bleeding volume was $(63.1 \pm 19.7) \mathrm{ml}$. These gave a total operation time of $(200.2 \pm 40.2)$ $\mathrm{min}$, and a total intraoperative bleeding volume of $(113.3 \pm 30.4) \mathrm{ml}$.

\section{Clinical outcomes}

The VAS score for low back pain of enrolled patients dropped from preoperative $(6.8 \pm 1.6)$ points to (1.4 \pm 1.0$)$ points at the final follow-up $(t=9.214, P=0.021)$, whereas the VAS score for leg pain declined from preoperative $(6.2 \pm 1.1)$ to $(1.0 \pm 0.5)$ points at the final follow-up $(t=10.321, P=0.015)$. Meanwhile, ODI decreased from preoperative $64.6 \pm 10.4 \%$ to $15.8 \pm 4.5 \%$ at the final follow-up $(t=12.213, P=0.011)$. Table 1 lists the details.

\section{Radiograph assessment}

According to the X-ray findings of enrolled patients, the coronal Cobb angle decreased from the preoperative value of $\left(20.3^{\circ} \pm 13.5^{\circ}\right)$ to $\left(5.5^{\circ} \pm 3.1^{\circ}\right)$ at the final follow-up $(t=7.123, P=0.024)$; LL increased from the preoperative value of $\left(25.8^{\circ} \pm 8.2^{\circ}\right)$ to $\left(39.3^{\circ} \pm 9.1^{\circ}\right)$ at the final follow-up $(t=-5.221, P=0.017)$; the intervertebral space height raised from the preoperative value of $(5.9 \pm 2.7) \mathrm{mm}$ to $(10.9 \pm 1.2) \mathrm{mm}$ at the final follow-up ( $t=-6.187, P=0.036)$; and SVA decreased from the preoperative value of $(42.3 \pm 16.5) \mathrm{mm}$ to $(25.1 \pm 10.9) \mathrm{mm}$ at the final follow-up $(t=-3.242, P=0.013)$. All differences were statistically significant. Figure 1 illustrates a typical case.

For the enrolled patients, 240 screws were inserted with the "O" arm CT navigation, and the excellent rate of intraoperative "O" arm 3D scanning was 96\% (230/240). Lumbar CT at 6 months postoperatively showed osseous fusion of all interbody fusion cages, with a fusion rate of $100 \%$.

\section{Complications}

Grade I/A incision healing was attained in all patients. No serious complications of nerve, blood vessel or abdominal organ injury werefound. Particularly, one patient developed anterior medial pain of the left thigh, two patients experienced a transient weakness of left hip flexion after surgery. They were not given special management, and all of them recovered during the follow-up period.

\section{Discussion}

With the ever intensified aging of China's population, the incidence of DS has been rising gradually, which has become a major cause of waist and leg pain among middle-aged and elderly populations. Currently, choice of DS treatment strategy and evaluation of relevant therapeutic efficacy have been the focus of spine surgeons. For patients with severe low back, leg pains and progressive aggravation of DS, which affect their quality of life and are not curable by conservative therapies, receiving active surgical treatment is needed. The aims of surgical treatment are to alleviate clinical symptoms, maintain spinal biomechanical stability and control the incidence of complications ${ }^{[24]}$. DS patients are characterized by 
multiple underlying diseases and weak surgical risk resistance since the majority of them are middleaged and elderly people. Ensuring the surgical safety is necessary while guaranteeing the therapeutic efficacy. Thus, it is important to reduce the surgical trauma and shorten the operation time. Conventional posterior osteotomy and orthopedic internal fixation have problems such as long operative duration, large intraoperative bleeding volume, high technical requirements and slow postoperative recovery ${ }^{[6-9]}$. Meanwhile, XLIF/DLIF combined with posterior internal fixation may cause damage to the psoas muscle and lumbar plexus, which can lead to rather high neurological complications as well ${ }^{[11-12]}$. OLIF, which enters obliquely through the natural gap between large abdominal vessels and psoas muscle from the anterior lateral side, dissects the psoas muscle bluntly, removes the intervertebral disc, restores the vertebral height, and decompresses stenotic spinal canal or nerve root canal indirectly, and can thereby correct the lateral kyphosis. Since the beginning of 2016, our hospital has been treating DS by OLIF combined with posterior " $\mathrm{O}$ " arm CT-navigated long segment internal fixation, which yielded satisfactory early clinical outcomes. With advantages like minimal invasiveness, accurate navigated screw insertion and high bone fusion rate, the surgical strategy can provide a new option for minimally invasive treatment of DS.

Apical vertebrae in DS are commonly seen in $\mathrm{L}_{2 / 3}, \mathrm{~L}_{3 / 4}$ position, while the segments exposed with OLIF are $L_{2} \sim L_{5}$, so OLIF can fully expose the surgical area, which has a unique advantage. With a surgical incision of approximately 3-4 cm, OLIF also has the advantages of small incision and low intraoperative bleeding. Silvestre et al. ${ }^{[25]}$ analyzed the data of 179 patients who underwent OLIF, and reported a mean bleeding volume of $57 \mathrm{ml}$ for a single segment. Fujibayashi et al . ${ }^{[26]}$ reported a mean blood loss of 17.6 $\mathrm{ml}$ in a single segment of OLIF.. In the present study, the 15 enrolled patients exhibited bleeding volume of merely $(50.2 \pm 10.7) \mathrm{ml}$ during the OLIF operation, so postoperative drainage tube placement was not needed. In the meanwhile, the posterior long segment internal fixation is implemented via the longissimus-multifidus approach to avoid damage to the paravertebral muscles. This procedure also has the small trauma, low bleeding advantages.

DS features degeneration of intervertebral discs in the scoliotic segments. The asymmetrical intervertebral stenosis causes lateral vertebral body slip, joint dislocation and ligamentum flavum hypertrophy, which can induce nerve traction and compression to result in corresponding clinical symptoms. OLIF procedure allows placement of a larger, wider interbody fusion cage than the posterior surgery, which can play an indirect decompression role. Sato et al. ${ }^{[14]}$ reported a $61 \%$ increase in the intervertebral space height, a $21 \%$ increase in the left side of intervertebral foramen area and a $39 \%$ increase in the right side of intervertebral foramen area after OLIF. Besides, VAS and ODI decreased markedly than before surgery, thus demonstrating a good indirect decompression effect of the surgery. For the patients enrolled in this study, the OLIF interbody fusion cage used was $45-55 \mathrm{~mm}$ in length and $12-16 \mathrm{~mm}$ in height. Besides, the intervertebral space height was $(5.9 \pm 2.7) \mathrm{mm}$ before surgery, and was $(10.9 \pm 1.2) \mathrm{mm}$ at the final follow-up, with an average increase of $84 \%$. The VAS and ODI at the final follow-up also improved remarkably than the preoperative values. Hence, OLIF combined with posterior "O" arm CT-navigated long segment internal fixation is effective in opening the intervertebral space and 
indirectly decompressing the spinal canal and intervertebral space, without the need for posterior spinal canal decompression, which avoids damage to the spinal dura mater and nerve roots.

Patients with DS often have coronal scoliosis, sagittal lumbar lordosis disappearance or segmental kyphosis, their coronal Cobb angle is not large, and main deformity is reduced lumbar lordosis ${ }^{[27]}$. OLIF is effective in correcting the coronal scoliosis and the sagittal imbalance. According to a report by Fujibayashi et al.$^{[26]}$, the lumbar lordosis angle of responsible vertebra increased by $4.5^{\circ}$ on average following OLIF as compared to the preoperative value. For the patients enrolled in this study, the interbody fusion cages were inserted in parallel by OLIF, and then the posterior long segment internal fixation was performed. At the final follow-up, the coronal Cobb angle decreased by $14.8^{\circ}$, LL increased by $13.5^{\circ}$, and SVA decreased by $17.2 \mathrm{~mm}$, thus correcting the coronal scoliosis and lumbar lordosis effectively and achieving sagittal balance.

Studies have found that the navigation aid can effectively enhance the accuracy of pedicle screw placement, shorten the screwing time, reduce the bleeding volume and lower the radiation exposure of both surgeons and patients ${ }^{[28-29]}$. For the patients enrolled in this study, 240 screws were placed via the "O" arm CT navigation. The excellent rate of intraoperative "O" arm 3D scanning was $96 \%$, and no screw insertion-related complications were noted.

OLIF is operated under direct vision through the natural gap anterior to psoas muscle. The psoas muscle is dissected bluntly while avoiding damage to the muscle and lumbar plexus. Damage to the spinal cord and nerve roots can be avoided by not entering the spinal canal. Meanwhile, retention of the anterior and posterior longitudinal ligaments can prevent vertebral instability, which effectively reduces the complications of lumbar plexus injury such as psoas muscle weakness, as well as numbness of inguinal region and anterior medial thigh. Among the 15 enrolled patients in this study, 1 patient developed anterior medial pain of left thigh, and 2 patients had weakness of left hip flexion postoperatively. All of them recovered during the follow-up period. The cause was analyzed to be excessive intraoperative traction. OLIF is capable of managing the responsible intervertebral discs fully, reducing the soft tissue adhesion of endplate and providing a preferable bone fusion environment. Moreover, after placement of the larger and wider interbody fusion cage, the surrounding ligament can be tightened to attain immediate stability, and the contact area of bone graft increases. Osseous fusion of all interbody fusion cages was found on the 6 months postoperative lumbar CT for the 15 patients in this study, with a fusion rate of $100 \%$.

In conclusion, the early clinical outcomes of the OLIF combined with posterior "O" arm CT-navigated long segment internal fixation for treatment of DS are satisfactory, with advantages like minimal invasiveness, accurate navigated screw insertion, high bone fusion rate and few complications, which can thus offer a new option for minimally invasive treatment of DS.

This study is a retrospective review, with small sample size, no control group and limited postoperative follow-up duration. A prolonged follow-up is needed to further observe and summarize the long-term 
outcomes and complications of the proposed surgical strategy.

\section{Abbreviations}

OLIF:Oblique lateral lumbar interbody fusion; CT: Computed tomography; MRI: Magnetic resonance imaging; LL:Lumbar lordosis; SVA:Sagittal vertical axis; ODI: Oswestry Disability index; VAS: Visual analogue scale; DS:Degenerative scoliosis; XLIF/DLIF:Extreme lateral interbody fusion or direct lumbar interbody fusion

\section{Declarations}

\section{Ethics approval and consent to participate}

The study protocol was approved by the Ethics Committee of Traditional Chinese Medical Hospital of Xiaoshan and the written informed consent was obtained from all patients.

\section{Consent for publication}

Not applicable

\section{Availability of data and materials}

We state that the data will not be shared since all the raw data are present in the figures included in the article.

\section{Competing interests}

The authors declare that they have no competing interests.

\section{Funding}

There is no funding in this article.

\section{Authors' contributions}

YL participated in the design of this study and performed the statistical analysis.drafted the manuscript. $\mathrm{RQ}$ participated in the study design and helped to draft the manuscript. HF and FH carried out the study and collected

important background information. CW collected the clinical data and follow-up details of this study. All authors read and approved the final manuscript.

\section{Acknowledgements}

None 


\section{References}

1. Hawasli $\mathrm{AH}$, Chang J,Yarbrough $\mathrm{CK}$, et al . Interpedicular height as a predictor of radicular pain in adult degenerative scoliosis[J]. Spine J, 2016,16(9):1070-1078.

2. Birknes JK, Harrop JS , White AP, et al. Adult degenerative scoliosis a review[J]. Neurosurgery, 2008, 63(3 Suppl):94-103.

3. Pellise F, Vila-Casademunt A, Ferrer M, et al. Impact on health related quality of life of adult spinal deformity(ASD) compared with chronic conditions[J]. Eur Spine J, 2015,24(1):3-11.

4. Aebi M . The adult scoliosis[J]. Eur Spine J,2005, 14(10):925-948.

5. Wang G, Hu J, Liu X. Et al. Surgical treatment for degenerative lumbar scoliosis:a meta analysis[J]. Eur Spine J, 2015,24(8):1792-1799.

6. Liu H, Yang C, Zheng Z, et al. Comparison of smith-petersen osteotomy and pedicle subtraction osteotomy for the correction of thoracolumbar kyphotic deformity in ankylosing spondylitis[J]. Spine(Phila Pa 1976), 2015,40(8): 570-579.

7. Yadla S , Ghobrial GM , Campbell PG , et al. Identification of complications that have a significant effect on length of stay after spine surgery and predictive value of 90 -day readmission rate[J]. $J$ Neurosurgery Spine, 2015,23(6):807-811.

8. Hua WB, Zhang YK, Gao Y, et al. Analysis of sagittal parameters in patients undergoing one- or twolevel closing wedge osteotomy for correcting thoracolumbar kyphosis secondary to ankylosing spondylitis[J]. Spine(Phila Pa 1976), 2017, 42(14):E848.

9. Charosky S, Guigui P, Blamoutier A , et al. Complications and Risk Factors of Primary Adult Scoliosis Surgery: A Multicenter Study of 306 Patients[J]. Spine(Phila Pa 1976), 2012, 37(8):693-700.

10. Ozgur B M , Aryan HE , Pimenta L, et al. Extreme Lateral Interbody Fusion (XLIF): a novel surgical technique for anterior lumbar interbody fusion[J]. The Spine Journal, 2006, 6(4):435-443.

11. Dahdaleh NS, Smith ZA, Snyder LA, et al. Lateral transpsoas lumbar interbody fusion: outcomes and deformity correction[J]. Neurosurg Clin N Am, 2014, 25(2):353-360.

12. Joseph JR, Smith BW, Marca FL, et al. Comparison of complication rates of minimally invasive transforaminal lumbar interbody fusion and lateral lumbar interbody fusion: A systematic review of the literature[J]. Neurosurgical FOCUS, 2015, 39(4):E4.

13. Lykissas MC, Aichmair A, Hughes AP, et al. Nerve injury after lateral lumbar interbody fusion: a review of 919 treated levels with identification of risk factors[J]. Spine J,2014,14(5):749-758.

14. Phan $\mathrm{K}$, maharaj $\mathrm{M}$, Assem $\mathrm{Y}$, et al. Review of early clinical results and complications associated with oblique lumbar interbody fusion(OLIF) [J]. J Clin Neurosci,2016,31:23-29.

15. Fujibayashi S, Hynes R A, Otsuki B , et al. Effect of Indirect Neural Decompression Through Oblique Lateral Interbody Fusion for Degenerative Lumbar Disease[J]. Spine(Phila Pa 1976), 2015, 40(3):E175-E182.

16. Sato J,Ohtori S, Orita A, et al. Radiographic evaluation of indirect decompression of mini-open anterior retroperitoneal lunbar interbody fusion: oblique lumbar interbody fusion for degenerated 
lumbar spondylolisthesis[J]. Eur Spine J, 2017, 26(3):671-678.

17. Jim M, Liu Z, Liu X, et al. Dose intraoperative navigation improve the accuracy of pedicle screw placement in the apical region of dystrophic scoliosis secondary to neurofibromatosis type I: comparison between O-arm navigation and free-hang technique[J]. Eur Spine J, 2016, 25(6):17291737.

18. Grelat M, Zairi F, Quidet M, et al. Assessment of surgeon radiation exposure during a minimally invasive TLIF: Comparison between fluoroscopy and O-arm system[J]. Neurochirurgie, 2015,61(4):255-259.

19. Gross Jb. Estimating allowable blood loss:corrected for dilution[J]. Anesthesiology,1953,58(3):277280.

20. Hornblow AR,Kidson MA. The visual analogue scale for anxiety:a validation study[J]. Aust N Z J Psychiatry,1976,10(4):339-341.

21. Fairbank JC,Pynsent PB. The oswestry disability index[J].Spine(Phila Pa 1976),2000,25(22):29402953.

22. Richter M, Mattes T, Cakir B. Computer-assisted posterior instrumentation of the cervical and cervicothoracic spine[J]. Eur Spine J,2004,13(1):50-59.

23. Siepe CJ, Stosch-Wiechert K, Heider F, et al. Anterior standalone fusion revisited: a prospective clinical, X-ray and CT investigation[J]. Eur Spine J,2015,24(4):838-851.

24. Cho SK,Bridwell KH,Lenke LG,et al. Major Complications in Revision Adult Deformity Surgery[J]. Spine(Phila Pa 1976), 2012, 37(6):489-500.

25. Silvestre C, Mac-Thiong JM, Hilmi R, et al. Complications and morbidities of mini-open anterior retroperitoneal lumbar interbody fusion:oblique lumbar interbody fusion in 179 patients[J]. Asian Spine J, 2012,6(2):89-97.

26. Fujibayashi S, Hynes RA, Otsuki B, et al. Effect of indirect neural decompression through oblique lateral interbody fusion for degenerative lumbar disease[J]. Spine (Phila Pa 1976), 2015, 40(3): E175E182.

27. Cho KJ, Kim YT, Shin SH, et al. Surgical treatment of adult degenerative scoliosis[J]. Eur Spine J, 2013, 8(3):371-381.

28. Meng XT, Guan XF, Zhang HL, et al. Computer navigation versus fluoroscopy-guided navigation for thoracic pedicle screw placement: a meta-analysis [J]. Neurosurg Rev, 2016,39(30):385-391.

29. Grelat M,Zairi F,Quidet $M$,et al. Assessment of the surgeon radiation exposure during a minimally invasive TLIF: Comparison between fluoroscopy and 0-arm system[J]. Neuro-Chirurgie, 2015, 61(4):255-259.

\section{Table 1}

Table 1 Comparison of preoperative and postoperative VAS and ODI between the two groups $\llbracket \mathrm{x} \pm \mathrm{s} \llbracket \operatorname{score} \square$ 


\begin{tabular}{lcccc}
\hline Item & Before surgery & After surgery & $t$ value & $P$ value \\
\hline VAS & & & & \\
Low back pain & $6.8 \pm 1.6$ & $1.4 \pm 1.0$ & 9.214 & 0.021 \\
Leg pain & $6.2 \pm 1.1$ & $1.0 \pm 0.5$ & 10.321 & 0.015 \\
ODI Overall score\%ロ & $64.6 \pm 10.4$ & $15.8 \pm 4.5$ & 12.213 & 0.011
\end{tabular}

\section{Figures}
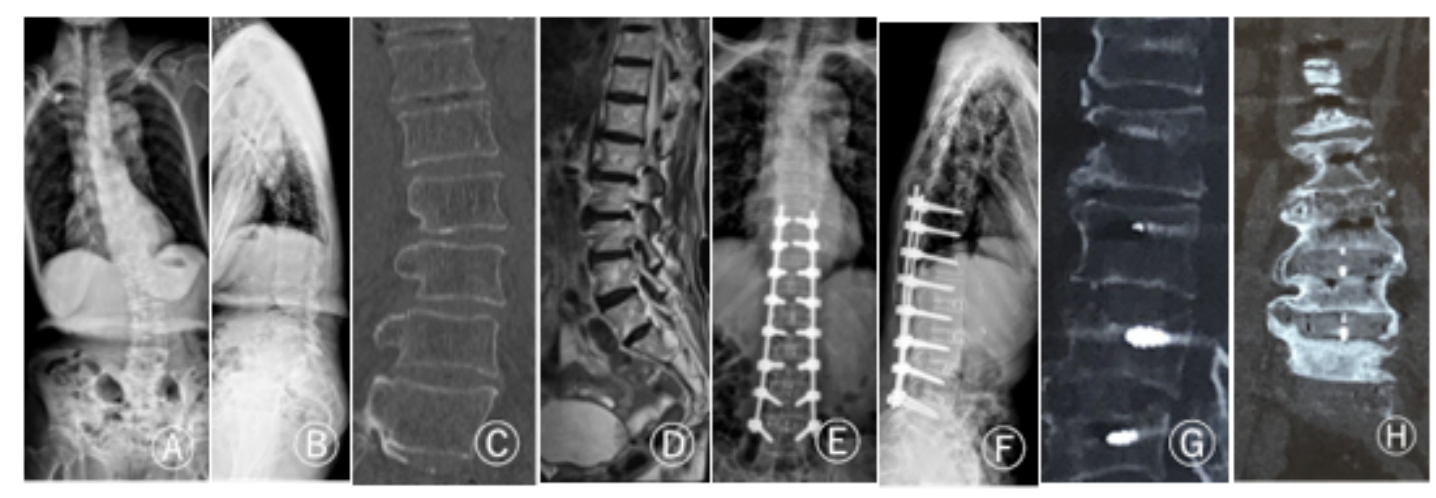

Figure 1

A 71-year-old female patient with degenerative scoliosis for 4 years, who underwent four-segment (L1/2, L2/3, L3/4, L4/5) OLIF combined with posterior T10-L5 internal fixation. A, B. Preoperative full-spinal PA \& LAT X-ray films in standing position revealed a Cobb angle of $25^{\circ}$, and a SVA of $46.6 \mathrm{~mm}$; C. Preoperative lumbar CT 3Dreconstruction showed that the scoliotic apex was located at L2/3; D.

Preoperative lumbar spine MRI showed lumbar scoliosis and lumbar spinal stenosis; E, F. Postoperative full-spinal PA \& LAT X-ray films revealed a postoperative Cobb angle of $5^{\circ}$, and a SVA of $22.7 \mathrm{~mm} ; \mathrm{G}, \mathrm{H}$. Six months postoperative reexamination of lumbar CT 3D reconstruction showed osseous fusion of the interbody fusion cage. Note: OLIF stands for oblique lateral lumbar interbody fusion 